\title{
ASSOCIAÇÃO ENTRE PATOLOGIAS, ESTRESSE OXIDATIVO E AGENTES ANTIOXIDANTES MAIS CONSUMIDOS - UM PANORAMA ATUAL
}

\section{ASSOCIATION BETWEEN PATHOLOGIES, OXIDATIVE STRESS AND MOST CONSUMED ANTIOXIDANT AGENTS - A CURRENT PANORAMA}

\author{
Marcela Rodrigues Moreira Guimarães; Carlos Magno Barros; Taissa Lima \\ Torres; Lucia Marques Vianna
}

\begin{abstract}
Resumo
É conhecido que a produção excessiva de substâncias activas de oxigénio reactivas o gene do factor de transcrição NFkappa B que activa enzimas de produção de eicosanóides cascata pró-inflamatória: AA-ciclo-oxigenase (COX-2) e 5-lipoxigenase (5 -LOX), resultando num aumento de PGE2, TXA2, LTB4, IL-6, TNF-alfa. Da mesma forma, substâncias reativas ao nitrogênio possuem dinâmica semelhante. Tal evento fisiopatológico parece comum a muitas patologias. A partir daí, uma série de investigações está sendo feita para identificar compostos da dieta com ação antioxidante e seus efeitos fisiológicos. Assim, este avaliar uma breve exposição da cena corrente, identificando antioxidantes fenólicos poli, vitaminas, e agentes, apreciar os resultados de ensaios de intervenção ou não, envolvendo ambos os animais experimentais e modelos clínicos. Diante de tal cenário, é evidente que já tem um número maior de tais alimentos funcionais que devem ser inseridos na dieta habitual, a fim de prevenir ou mesmo parar a progressão de doenças que ocorrem com o estresse oxidativo.
\end{abstract}

Palavras-chave: Estresse oxidativo, antioxidantes, vitaminas, agentes poli fenólicos, inflamação.

\begin{abstract}
It is known that the excessive production of reactive oxygen species activates the gene transcription factor NFKappa B that activates the enzymes of the production cascade of pro-inflammatory eicosanoids: AA-cyclooxygenase (COX-2) and 5lipoxygenase (5 -LOX), resulting in an increase of PGE2, TXA2, LTB4, IL-6, TNFalpha. In the same way, reactive nitrogen species have similar dynamics. Such physio pathological event seems common to many pathologies. From there, a series of investigations are being done to identify compounds of the diet with antioxidant action and their physiological effects. Thus, this review gives a brief overview of the current situation, identifying antioxidants, vitamins and poly phenolic compounds, appreciating the results of intervention trials or not, involving both experimental and clinical animal models. In view of such a scenario, it is evident that there is already a greater number of functional foods that must be inserted into the habitual diet for the purpose of prevention or even in the arrest of the progression of disorders that present with oxidative stress.
\end{abstract}

Keywords: Oxidative stress, antioxidants, vitamins, polyphenolic agents, inflammation 


\section{INTRODUÇÃO}

É sabido que a produção excessiva de substâncias reativas ao Oxigênio: anion superóxido, (O2 --), peróxido de hidrogênio (H2O2) e radical hidroxila, (HO-) coletivamente chamadas de ROS, ativa o fator de transcrição gênica NFKappa B, que ativa as enzimas da cascata de produção de eicosanoides pró-inflamatórios: a AA-ciclooxigenase (COX-2) e a 5-lipooxigenase (5-LOX), resultando em aumento de PGE2, TXA2, LTB4, IL-6, TNF-alfa. Da mesma forma, substâncias reativas ao Nitrogênio (RNS), como NO e ONOO-, tem dinâmica similar. Ao mesmo tempo, durante o processo inflamatório, as células fagocíticas, principalmente neutrófilos e macrófagos, realizam um rápido consumo de oxigênio, processo conhecido como respiratory burst. Esse processo é uma das fontes de formação de superóxido, peróxido de hidrogênio, radical hidroxila, ácido hipocloroso e peroxinitrito. Esta ativação do sistema imunológico portanto desencadeia mais produção de ROS e RNS gerando um ciclo vicioso entre estresse oxidativo e processo inflamatório'.

Tal evento fisiopatológico parece comum à muitas patologias como a hipertensão arterial, o diabetes mellitus e outras de origem neurológica, osteomuscular e neoplasias em geral. A partir daí uma série de investigações vem sendo feitas para identificar compostos da dieta com ação antioxidante e seus efeitos fisiológicos em modelos animais sujeitos ao estresse oxidativo e ao processo inflamatório. Assim, esta revisão faz uma breve exposição do panorama atual, identificando os agentes antioxidantes: vitaminas e compostos polifenólicos, apreciando os resultados de ensaios de intervenção ou não, envolvendo tanto modelos animais experimentais quanto clínicos.

\section{DESENVOLVIMENTO}

Ensaios com modelos animais portadores de hipertensão arterial espontânea (SHR) e com modelos animais hipertensos e propensos ao acidente vascular encefálico (SHRSP) revelam que esses animais apresentam altos níveis de marcadores de estresse oxidativo e de processo inflamatório². A atenuação 
deste quadro, vem ao longo dos anos, sendo o principal objetivo de ensaios que usam compostos de reconhecido poder antioxidante in vitro, como o alfatocoferol$^{3-6}$, o beta-caroteno ${ }^{7}$, dentre outros não comumente citados como antioxidantes, como por exemplo as vitaminas $\mathrm{D}^{8,9}, \mathrm{~K}^{10}$ e vitaminas do complexo $\mathrm{B}$ : $\mathrm{B} 2^{11,12} \mathrm{~B} 6$ e B9 ${ }^{13}$. Em linhas gerais, os ensaios supracitados, demonstraram importante redução da pressão arterial sistólica, dos biomarcadores de estresse oxidativo e de processo inflamatório além da correção do lipidograma, das alterações histopatológicas em músculo liso visceral e vascular e da hemodinâmica dos animais SHR e SHRSP. Na linhagem SHRSP o tratamento com as vitaminas supracitadas também resultou em melhor resposta à testes sensório-motores e cognitivos, revelando atividade neuroprotetora.

Por outro lado, ensaios com grupos populacionais e/ou clínico randomizados nem sempre são tão conclusivos. Por exemplo, uma recente revisão que teve como objetivo associar o consumo de vitaminas antioxidantes da dieta à marcadores de aterosclerose em mulheres algerianas, somente encontrou tal associação com as vitaminas $\mathrm{C}$ e $\mathrm{E}$, embora tenham rastreados outras vitaminas. Porém, ainda que estimulados pelos positivos resultados, os autores concluíram, enfaticamente pela necessidade de mais estudos antes da adoção de prescrição de vitaminas para prevenção de doenças cardiovasculares ${ }^{14}$.

Entretanto, Shahidi e Camargo (2016) $)^{15}$ apresentaram uma revisão que, reunindo dados de 1978 até 2016 e explorando as bases de dados Pubmed, Scielo, Scopus, Web of Science e Google Scholar, revelou serem os efeitos protetores do tocoferol numerosos e abrangentes no caso de diferentes tipos de câncer, diabetes, doença cardiovascular e obesidade. Nesta revisão, os autores destacaram dois estudos na área cardiovascular que envolveram intervenção com alfa-tocoferol. Um deles utilizou $400 \mathrm{UI} /$ dia o que levou à redução da LDL-oxidada ${ }^{16}$ e o outro estudo, que foi uma metaanálise, concluiu que doses de alfa-tocoferol variando de 400 a $800 \mathrm{UI} /$ dia durante oito semanas promoveram redução de $20 \%$ na ocorrência de infarto do miocárdio e, também concluíram, que doses menores que $400 U \mathrm{U} /$ dia não tiveram qualquer efeito ${ }^{17}$.

Por outro lado, não é possível desconsiderar que alguns trabalhos alertam para o risco do consumo excessivo de vitaminas. Em relação a isso, Amaya- 
Farfan et al. $(2001)^{18}$, descreveram, em interessante publicação, as novas propostas sobre recomendações nutricionais para antioxidantes e, discutiram vários tópicos que abordam desde as modificações destas recomendações a luz das evidências relativas aos pressupostos efeitos benéficos e os riscos da suplementação destes nutrientes. Os autores citam, pelo menos, dois trabalhos que encontraram aumento da mortalidade pelo câncer de pulmão em indivíduos que receberam suplementação de beta-caroteno.

Madhun et al. (2010) ${ }^{19}$, também apresentaram uma revisão de estudos longitudinais, muitos dos quais multicêntricos, com grande amostragem e follow-up de longa duração, e analisaram os efeitos anti e pró-oxidantes de vitaminas e também de minerais, e igualmente relataram ocorrências de efeitos prejudiciais incluindo taxa de mortalidade, quando do uso de megadoses. No que se refere a isso, acrescenta-se também recente publicação, que associa à ingestão aumentada de retinol o carcinoma de pulmão em homens ${ }^{20}$. Da mesma forma, é amplamente conhecido que o tocoferol, ao atuar como antioxidante, também se torna pró-oxidante, quando forma a tocoferrilquinona, o que justifica a prescrição concomitante de vitamina $E$ e ácido ascórbico. É interessante também observar que tanto os ensaios clínicos quanto os experimentais revelam eficácia das vitaminas antioxidantes somente mediante o emprego de doses suprafisiológicas, o que aumenta a necessidade de um acompanhamento bastante criterioso observando cuidadosamente os riscos advindos de tal prescrição.

Agentes polifenólicos

Os polifenóis podem neutralizar os radicais livres através de diversos mecanismos: pela doação de elétrons e/ou desativando espécies ativas e precursores de radicais livres ou atuando como agentes quelantes de metais como o ferro, por exemplo, inibindo oxidases. Ensaios mais recentes, também sugerem que polifenóis possam inibir fatores de transcrição gênica como o NFKappaB e aumentar a expressão de genes que codificam proteínas como a superóxido dismutase e a glutationa peroxidase ${ }^{21}$. Como resultado final, o efeito fisiológico de polifenois traduz-se em redução de biomarcadores de estresse oxidativo e de processo inflamatório, diminuição da oxidação da LDL,redução da aterosclerose, dentre outros benefícios. 
Em relação a isso, ensaios observacionais com população de origem nipônica, revelaram a associação entre alta ingestão de compostos polifenólicos, tendo como fonte alimentar predominante o chá verde, e baixa prevalência de hipertensão arterial e fatores preditivos de doença vascular ${ }^{22}$. Recentemente, a ingestão de chá verde, em média 4 xicaras, vem sendo recomendada em livros texto, como estratégia para redução do fator de risco de acidente vascular encefálico ${ }^{23}$.

A ingestão de suco de uva, rico em resveratrol, também vem sendo associada à baixa oxidação de proteínas, e, sendo recomendada na prevenção de doenças cardiovasculares ${ }^{24}$. Mais recentemente, Fiorelli (2017) ${ }^{25}$ demonstrou ação benéfica da fruta Romã, também importante fonte de compostos fenólicos. Segundo o autor, animais submetidos à suplementação da dieta com farinha liofilizada de Punica granatum, apresentaram: redução da pressão arterial, diminuição de marcadores de estresse oxidativo e de processo inflamatório e obtiveram melhor resposta aos testes de cognição e redução em número e severidade dos ataques isquêmicos transitórios.

$\mathrm{Na}$ realidade, a associação entre flavonoides e a redução de processo inflamatório, também foi previamente citada em situações que possam envolver injúria neuronal. Em relação a isso, Vafeiadou et al. $(2009)^{26}$, também observaram em população feminina, acompanhada por dez anos, uma redução do risco de depressão naquelas que ingeriram duas ou mais porções de frutas cítricas por dia. Spencer $(2009)^{27}$ atribuiu aos flavonoides melhora do fluxo sanguíneo, função antiinflamatória e neuroprotetora. Ramesh et al. (2010) ${ }^{28}$ confirmaram os achados anteriores e sugeriram que os flavonoides atuem como substâncias bioativas protetoras da saúde mental tendo efeito sobre a cognição, memória e coordenação.

Destaca-se também, uma interessante revisão sobre este tema, que citou uma série de ensaios que usaram a técnica de ressonância magnética para explorar os efeitos do cacau enriquecido com flavonoides sobre o fluxo sanguíneo cerebral. Segundo os autores, o uso de $150 \mathrm{mg}$ deste produto por cinco dias, promoveu melhor oxigenação cerebral. Relataram também, que uma dose única de $450 \mathrm{mg}$ de cacau enriquecido com flavonoides foi capaz de aumentar o fluxo 
sanguíneo na massa cinzenta, e, finalizaram suas conclusões reforçando o "potencial benéfico dos flavonoides no tratamento das desordens vasculares, demência e acidente vascular encefálico, do qual milhares de pessoas poderiam se beneficiar"29.

Além das bebidas supracitadas, o café também ganha destaque nas investigações acerca de suas prováveis propriedades benéficas, especialmente devido ao seu alto conteúdo de compostos fenólicos, ácido clorogênico e vitamina B3. Na realidade, imediatamente após a ingestão de café a pressão arterial sobe, a resistência à insulina aumenta bem como a intolerância à glicose, mas a ingestão regular de café não parece afetar a pressão arterial ou a resistência à insulina. Adicionalmente, Groso $\mathrm{G}$ et al. (2017) ${ }^{30}$, em uma ampla revisão também confirmaram os efeitos benéficos do café na saúde humana, sendo associado à redução do risco de doença cardiovascular, diabetes do tipo II, diversos tipos de neoplasias, e até Mal de Parkinson. Por outro lado, no que se refere a sua atuação sobre a memória, nem sempre é encontrada unanimidade entre os achados. Em relação a isso, recente revisão sistemática que incluiu acima de 400000 participantes não confirmou os supostos efeitos do café sobre a memória ${ }^{31}$.

Ensaios que visam identificar estimativas de consumo de específicos antioxidantes e suas fontes alimentares também apontam para uma forte inversa associação com a ocorrência de Diabetes do tipo II e Síndrome Metabólica. Dentro deste contexto, Guo X et al. $(2016)^{32}$ relataram que o alto consumo de antocianinas foi capaz de contribuir na prevenção do Diabetes Tipo II. Nesse estudo que envolveu mais de 200.000 participantes, o alimento fonte foi a cereja. Em 2017, Guo X et al. ${ }^{33}$, também demonstraram uma correlação inversa entre o consumo de maçãs e pêras e o risco de Diabetes Melllitus do Tipo II, chegando a sugerir que o risco poderia ser reduzido a $18 \%$. O referido trabalho reuniu mais de 220.000 participantes.

Em recente estudo clinico randomizado, Chew B et al. (2018) ${ }^{34}$ também associaram dieta hipocalórica e o alto consumo de bebida rica em polifenois à atenuação do processo inflamatório e a regulação do metabolismo glicídico em individuos com sobrepeso. Nesse estudo, a fruta usada no suco foi o mirtilo vermelho. Uma vez mais, recente metaanálise, revelou que a alta ingestão de 
flavonóides e suas subclasses (antocianinas e isoflavonas), também está associada à redução do risco de desenvolvimento do Diabetes tipo $\|^{35}$. Yao $Z$ et al. $(2018)^{36}$, também atribuíram à quercetina, a redução de ocorrência de Diabetes Tipo II em adultos chineses que habitualmente consomem chá verde, maçã e laranja.

No que se refere às fontes alimentares de antioxidantes, Locatelli $\mathrm{G}$ et al. (2014) $)^{37}$ assinalaram que frutas, hortaliças e cereais são as fontes de maior teor de compostos fenólicos nas dietas brasileiras, sendo que dentre as frutas destacase o açaí. Adicionalmente, recente investigação, revelou que o café, o mate, o açaí e feijões são as mais presentes na dieta brasileira ${ }^{38}$. Em trabalhos prévios, as especiarias também foram citadas como importantes fontes de antioxidantes na culinária brasileira ${ }^{39}$.

No que se refere às características de consumidores de antioxidantes, estudos observacionais, ainda que escassos, revelaram possível diferença de consumo entre sexos, sendo que homens apresentariam maior ingestão de vitamina E, zinco, cobre e magnésio do que mulheres. Entretanto, ambos consumiram níveis de Vitamina E e Cobre abaixo do recomendado e Magnésio acima do recomendado ${ }^{40}$. Em um estudo transversal com coleta de dados secundários do consumo alimentar que utilizou o banco de dados do IBGE dos anos 2008/ 09, Tureck $C$ et al. (2017) ${ }^{41}$, também relataram baixo consumo de vitamina $E$ mas também de vitamina $C$, sendo os níveis inadequados em $72 \%$ da amostra total. Entretanto reportaram que os indivíduos obesos apresentaram os maiores níveis de inadequação.

Resumidamente, a literatura tenta explicar a aparente contradição dentre os ensaios clínicos que usam antioxidantes naturais, incluindo vitaminas, tomando por base a dificuldade de biodisponibilidade desses agentes antioxidantes, sua específica farmacocinética e farmacodinâmica e à produção de ROS que é de certa forma generalizada no corpo humano e principalmente à limitação da ação desses agentes antioxidantes no tecido alvo ${ }^{42}$. Embora os autores reconheçam como promissoras algumas vitaminas e polifenólicos, também sugerem que a formulação destes agentes em nanopartículas poderá prolongar a atividade destes antioxidantes e permitir sua ação em tecidos que estejam sendo alvos de ROS 
e/ou reativas ao nitrogênio, o que permitirá o uso de antioxidantes na conduta clínica preventiva e/ou curativa do estresse oxidativo, além de evitar o uso destes agentes em doses suprafisiológicas ${ }^{43}$.

\section{Conclusões}

Ainda que não tenha sido encontrada unanimidade entre os ensaios experimentais, clinico-randomizados ou nos estudos observacionais, aqui apresentados, aqueles trabalhos, independentemente do design, que evidenciaram efeitos benéficos na suplementação de vitaminas antioxidantes tiveram como ponto comum o fato de que tais achados apenas ocorreram com doses suprafisiológicas.

No que se refere aos agentes polifenólicos, a falta de consenso também foi presente, tornando-se um impeditivo na indicação destes nutrientes com finalidade curativa nas doenças que cursam com estresse oxidativo. Por outro lado, a literatura, de forma genérica, continua reforçando a importância de aumentar o consumo diário de frutas e hortaliças, fontes de antioxidantes, na manutenção da saúde e na prevenção de doenças, e recomenda a realização de mais ensaios clinico-randomizados com a finalidade de alcançar, em futuro próximo, uma definição acerca das doses apropriadas para cada caso.

Da mesma forma, esta revisão mostrou uma série de alimentos que através de sua composição em bioativos, podem contribuir preventivamente e/ou atuar como coadjuvantes no tratamento das desordens que cursam com estresse oxidativo. Em relação a isso, ficou evidente que os trabalhos que mostraram tal associação, usaram como parte das ferramentas de seus ensaios: o recordatório alimentar, que foi de 24 horas e/ou de três dias, o questionário de frequência alimentar, reunindo mais de cem itens em alguns estudos e acompanhado de pranchas ilustrativas de utensílios o que contribuiu sobremaneira na qualidade metodológica.

Em vista de tal panorama, resumidamente aqui apresentado, fica evidente que já se dispõe de um maior número de alimentos ditos funcionais que devem ser inseridos na dieta habitual com finalidade de prevenção ou mesmo na detenção da 
progressão de desordens que cursam com estresse oxidativo. Além disso, reforçamos a importância de uma prescrição com acompanhamento criterioso quando da suplementação isolada de nutrientes com a finalidade de modular o estresse oxidativo, haja vista a efetividade desta conduta apenas com doses suprafisiológicas.

\section{Bibliografia}

[1] Montera VSP. Benefits of antioxidants nutrients and their cofactors for oxidation stress and inflammation control in heart failure. Ver. SOCERJ. 2007; 20 (1): 20-27.

[2] Fiorelli S, França CF, Basílio-de-Oliveira CA et al. Oxidative stress unify physiopathological mechanism between severe hypertension and diabetes. Research \& Reviews: Neuroscience. 2017; 1 (3): 3-7.

[3] Costa V, Vianna LM, Aguila MB et al. Alpha-tocopherol supplementation: favorable effects on blood pressure, blood viscosity and cardiac remodeling of spontaneously hypertensive rats. J Nutr Biochem. 2005; 16 (4): 251-256.

[4] Guimarães MRM, Murad LB, Paganelli, A et al. Alpha-tocopherol treatment is able to modulate heart and kidney histopathological features of shrsp. Curr Top Nutraceut R. 2014; 12 (1-2): 19-24.

[5] Murad LB, Guimarães MRM, Paganelli A et al. Alpha-tocoherol in brain tissue preservation of spontaneously hypertensive rats stroke-prone. J Physiol Biochem. 2014; 70 (1): 49-60.

[6] Guimarães MRM, Murad LB, Paganelli A et al. Effects of alpha-tocopherol associated to lovastatin on brain tissue and memory function in SHRSP. Physiol Behav. 2015; 149: 303-309.

[7] Fiorelli S, Vianna LM, Basílio-de-Oliveira CA et al. The effects of supraphysiological supplementation of b-carotene in spontaneously hypertensive rats (SHR and SHR-sp). Rev. Col. Bras. Cir. 2014; 41(5): 351-355.

[8] Vianna LM, Paiva ACM, Paiva TB. Treatment with Vitamin D lowers blood pressure of Spontaneously Hypertensive Rats. Genetic Hypertension. 1992; 218: 589-591.

[9] Borges A, Feres T, Vianna LM et al. Recovery of an impaired $\mathrm{K}+$ channels in mesenteric arteries from spontaneously hypertensive rats by prolonged treatment with cholecalciferol. Br J Pharmacol. 1999; 126 (3): 772-778.

[10] Cavargere VA, Guimarães MRM, Murad LB et al. Effects of Vitamin K1 Supplementation on the Risk Factors for Stroke in Spontaneously Hypertensive Rats Stroke Prone (SHR-sp). Braz. Arch. Biol. Technol. 2016; 59: 4-9.

[11] França CF, Vianna LM. Riboflavina no controle da hipertensão e no acidente vascular encefálico. Neurociências. 2009; 5: 40-45.

[12] França CF, Vianna LM. Effectiveness of $B$ vitamins on the control of hypertension and stroke events of SHRSP rats. J Diet Suppl. 2010; 7 (1): 71-77.

[13] Perez S, Vianna LM. Favorable effect of pyridoxine and folic acid supplementation of stroke-prone rats. Arch Neurocien (Mex). 2005; 10 (3): 146149.

[14] Diaf M, Khaked MB. Associations Between Dietary Antioxidant Intake and

Revista Inova Saúde, Criciúma, vol. 12, n. 2, julho. 2021. ISSN 2317-2460 
Markers of Atherosclerosis in Middle-Aged Women From North-Western Algeria. Front Nutr. 2018; 5 (29).

[15] Shahidi F, Camargo AC. Tocopherols and Tocotrienols in Common and Emerging Dietary Sources: Occurrence, Applications, and Health Benefits. Int J Mol Sci. 2016; 17 (10).

[16] Jialal I, Fuller C, Huet BA. The effect of alpha-tocopherol supplementation on LDL oxidation. A dose-response study. Arterioscler Thromb Vasc Biol. 1995; 15 (2): 190-198.

[17] Loffredo L, Perri L, Di Castelnuevo A et al. Supplementation with vitamin $\mathrm{E}$ alone is associated with reduced myocardial infarction: a meta-analysis. Nutr Metab Cardiovasc Dis. 2015; 25 (4): 354-63.

[18] Amaya-Farfan J, Domene SMA, Padovani RM. DRI: Síntese comentada das novas propostas sobre recomendações nutricionais para antioxidantes. Rev. Nutr. 2001; 14 (1): 71-78.

[19] Soni MG, Thurmond TS, Miller ER et al. Safety of Vitamins and Minerals: Controversies and Perspective. Toxicol Sci. 2010; 118 (2): 348-355.

[20] Narita S, Saito E. Dietary consumption of antioxidant vitamins and subsequent lung cancer risk: The Japan Public Health Center-based prospective study. International Journal of Cancer. 2018; 142 (12): 2441-1460.

[21] Sefora-Sousa M, De Angelis Pereira MC. Mecanismos moleculares de ação anti-inflamatória e antioxidante de polifenóis de uvas e vinho tinto. Rev. Bras. Plantas Med. 2013; 15 (40): 617-626.

[22] Faria F, Santos RS, Vianna LM. Consumo de Camellia sinensis em população de origem oriental e incidência de doenças crônicas. Rev. Nutr. 2006; 19 (2): 275279.

[23] Bonow RO, Mann DL, Zipes DP, Libby P, Braunwald E. Braunwald - Tratado de Doenças Cardiovasculares. Rio de Janeiro: Elsevier, 2017.

[24] O'Byrne DJ, Devaraj S, Grungy SM et al. Comparison of the antioxidant effects of Concord grape juice flavonoids alpha-tocopherol on markers of oxidative stress in healthy adults. Am J Clin Nutr. 2002; 76 (6):1367-1374.

[25] Fiorelli S. Efeitos da suplementação de casca liofilizada de Punica granatum a ratos SHRSP. [Tese]. [Rio de Janeiro]: Faculdade de Medicina, Universidade Federal do Estado do Rio de Janeiro; 2017.

[26] Vafeiadou K, Vauzour D, Lee HY et al. The citrus flavanone naringenin inhibits inflammatory signalling in glial cells and protects against neuroinflammatory injury. Arch Biochem Biophys. 2009; 484 (1): 100-109.

[27] Spencer JP. Flavonoids and brain health: multiple effects underpinned by common mechanisms. Genes Nutr. 2009; 4 (4): 243-250.

[28] Ramesh B, Rao S, Prakasam A et al. Neuronutrition and Alzheimer's Disease. J Alzheimers Dis. 2010; 19 (4): 1123-1139.

[29] Patel AK, Rogers JT, Huang X. Flavanols, Mild Cognitive Impairment, and Alzheimer's Dementia. Int J Clin Exp Med. 2008; 1 (2): 181-191.

[30] Grosso G, Godos J, Galvano F et al. Coffee, Caffeine, and Health Outcomes: An Umbrella Review. Annu Rev Nutr. 2017; 37: 131-156.

[31] Zhou A, Taylor AE, Karhunen $V$ et al. Habitual coffee consumption and cognitive function: a Mendelian randomization meta-analysis in up to 415.530 participants. Sci Rep. 2018; 8 (1): 7526-7535. 
[32] Guo X, Yang B, Tan J et al. Associations of dietary intakes of anthocyanins and berry fruits with risk of type 2 diabetes mellitus: a systematic review and metaanalysis of prospective cohort studies. Eur J Clin Nutr. 2009; 70 (12): 1360-1367.

[33] Guo XF, Yang B, Tang $\mathrm{J}$ et al. Apple and pear consumption and type 2 diabetes mellitus risk: a meta-analysis of prospective cohort studies. Food Funct. 2017; 8 (3): 927-934.

[34] Chew B, Mathison B, Kimble L et al. A randomized chronic consumption of a low calorie, high polyphenol cranberry beverage attenuates inflammation and improves glucoregulation and $\mathrm{HDL}$ cholesterol in healthy overweight humans controlled trial. Eur J Nutr. 2019; 58 (3): 1223-1235.

[35] $\mathrm{Xu} \mathrm{H}$, Luo J, Huang $\mathrm{J}$ et al. Flavonoids intake and risk of type 2 diabetes mellitus: A meta-analysis of prospective cohort studies. Medicine (Baltimore). 2018; 97 (19): 686.

[36] Yao Z, Gu Y, Zhang $Q$ et al. Estimated daily quercetin intake and association with the prevalence of type 2 diabetes mellitus in Chinese adults. Eur J Nutr. 2019; 58 (2): 819-830.

[37] Locateli G, Tureck $C$ et al. Identificação dos alimentos fontes de antioxidantes da dieta brasileira. Anais do SEPE - Seminário de Ensino, Pesquisa e Extensão da UFFS. 2014; 4 (1) [acesso em 19 julho de 2018]. Disponível em https://periodicos.uffs.edu.br/index.php/SEPE-UFFS/article/view/1165.

[38] Torres T, Farah A. Coffee, maté, açaí and beans are the main contributors to the antioxidant capacity of Brazilians diet. Eur J Nutr. 2017; 56 (4): 1523-1533.

[39] Del Ré PV, Jorge N. Especiarias como antioxidantes naturais: aplicações em alimentos e implicação na saúde. Rev. Bras. Plantas Med. 2012; 14 (2): 389-399. [40] Oliveira IP, Bergjohamn P, Adami FS et al. Consumo de antioxidantes entre praticantes e não praticantes de atividade física. RBNE. 2017; 11 (64): 428-436.

[41] Tureck C, Locateli G, Correa VG et al. Avaliação da ingestão de nutrientes antioxidantes pela população brasileira. Rev. Bras. Epidemiol. 2017; 20 (1): 30-42. [42] Liu Z, Ren Z, Zhang J et al. Role of ROS and Nutritional Antioxidants in Human Diseases. Front Physiol. 2018; 9: 477-482.

[43] Nash KM, Ahmed S. Nanomedicine in the ROS-Mediated Pathophysiology: Applications and Clinical Advances. Nanomedicine. 2015; 11 (8): 2033-2040. 